\title{
Occult Breast Cancer
} Presenting as Metastatic Adenocarcinoma of Unknown Primary: Clinical Presentation, Immunohistochemistry, and Molecular Analysis

\author{
Jue Wang ${ }^{\mathrm{a}}$ Geoffrey Talmon ${ }^{\mathrm{b}}$ Jordan H. Hankins ${ }^{\mathrm{c}}$ \\ Charles Enke ${ }^{\mathrm{d}}$ \\ ${ }^{a}$ Division of Oncology and Hematology, Department of Internal Medicine, and \\ Departments of ${ }^{b}$ Pathology and Microbiology, ${ }^{\mathrm{c}}$ Radiology, and ${ }^{\mathrm{d}}$ Radiation \\ Oncology, University of Nebraska Medical Center, Omaha, Nebr., USA
}

\section{Key Words}

Carcinoma of unknown primary - Triple-negative breast cancer . Immunohistochemistry · Molecular profiling

\begin{abstract}
We report a rare presentation of a 66-year-old female with diffuse metastatic adenocarcinoma of unknown primary involving liver, lymphatic system and bone metastases. The neoplastic cells were positive for CK7 and OC125, while negative for CK20, thyroid transcription factor 1, CDX2, BRST-2, chromogranin, synaptophysin, estrogen receptor (ER), progesterone receptor (PR), and human epidermal growth factor receptor 2 (HER2/neu). Fluorescence in situ hybridization showed no amplification of the HER2/neu gene. Molecular profiling reported a breast cancer origin with a very high confidence score of $98 \%$. The absence of immunohistochemistry staining for ER, PR, and HER2/neu further classified her cancer as triple-negative breast cancer. Additional studies revealed high expression levels of topoisomerase (Topo) I, androgen receptor, and ribonucleosidediphosphate reductase large subunit; the results were negative for thymidylate synthase, Topo II- $\alpha$ and 06-methylguanine-DNA methyltransferase. The patient was initially treated with a combination regimen of cisplatin and etoposide, and she experienced a rapid resolution of cancer-related symptoms. Unfortunately, her therapy was complicated by a cerebrovascular accident (CVA), which was thought to be related to cisplatin and high serum mucin. After recovery from the CVA, the patient was successfully treated with second-line chemotherapy based on her tumor expression profile. We highlight the role of molecular
\end{abstract}


profiling in the diagnosis and management of this patient and the implication of personalized chemotherapy in this challenging disease.

\section{Introduction}

Carcinoma of unknown primary (CUP) is a biopsy-proven epithelial malignancy for which the anatomic site of origin remains unidentified after an intensive search. CUP is one of the ten most frequently diagnosed cancers worldwide, accounting for approximately $3-5 \%$ of all cancer cases $[1,2]$.

We report a rare presentation of a 66-year-old female, with diffuse metastatic adenocarcinoma of unknown primary involving the liver, lymphatic system and spine, who was successfully treated with chemotherapy based on her tumor expression profile. We highlight the role of molecular profiling in the diagnosis and management of this patient.

\section{Case Report}

Clinical, Radiologic, and Laboratory Findings

A 66-year-old woman was hospitalized in April 2010 for generalized weakness and a 50-pound weight loss. Her history was notable for cholecystectomy and hysterectomy. She had a 15-pack/year smoking history but had quit six years previously. Her family history was negative for any cancer. She was not on any regular medications and had no allergies. The initial physical examination showed a cachectic female with a large left neck mass and enlarged liver. Breast and pelvic examinations revealed no suspicious lesions. Laboratory studies revealed the following values: aspartate aminotransferase $260 \mathrm{U} / \mathrm{l}$ [normal range (NR) 11-66], alanine aminotransferase $123 \mathrm{U} / \mathrm{l}$ (NR 15-46), alkaline phosphatase 1,372 U/l (NR 38-126), lactate dehydrogenase $783 \mathrm{U} / \mathrm{l}$ (NR 313-618), and albumin $2.8 \mathrm{~g} / \mathrm{dl}$ (NR 3.6-5.0). In addition, the patient had elevated serum levels of CA 15-3: 642 U/ml (NR 0-31), CA 19-9: $128 \mathrm{U} / \mathrm{ml}$ (NR 0-35), CA 125: 4,533 U/ml (NR 0-35), and CA 27.29: 1,262 U/ml (NR 0-48). Computed tomography (CT) scans of the neck, chest, abdomen and pelvis showed left neck, supraclavicular area, thoracic inlet, gastrohepatic and retroperitoneal lymphadenopathy, along with left lower lobe pulmonary nodules, and diffuse liver metastases. Needle biopsies of one of the liver lesions were performed and demonstrated a poorly differentiated adenocarcinoma. Extensive workup searching for a primary tumor, including panendoscopies and mammogram examination, was negative. A fluorodeoxyglucose positron emission tomography (FDG-PET) study demonstrated multiple hypermetabolic lesions involving the left neck, superior mediastinal mass, and multiple segmental liver and skeletal structures. Magnetic resonance imaging (MRI) of the abdomen revealed multiple liver masses, with the largest measuring approximately $8 \times 5 \mathrm{~cm}$ in hepatic segment 4 . These masses were hyperintense on enhanced $\mathrm{T}_{2}$-weighted sequence. Additionally, there were enhancing lesions compatible with bone metastases in T11, T12, L1 and L3 (fig. 1a).

The patient was initially treated with a combination regimen of cisplatin and etoposide; she had a rapid resolution of cancer-related symptoms. In addition, zoledronic acid (Zometa ${ }^{\circledR}$ ) was initiated for her metastatic bone disease. Unfortunately, her therapy was complicated by a cerebrovascular accident (CVA) 4 weeks after the initiation of chemotherapy. An MRI of the brain showed multifocal small infarcts in the right cerebral hemisphere involving middle cerebral artery distribution, the watershed area, and to a lesser degree, posterior cerebral artery distribution. Despite the dismal MRI findings, she eventually recovered from the CVA without any residual weakness. Six weeks after the CVA, chemotherapy was restarted and changed to FOLFIRI based on her tumor profile. A restaging PET-CT in March 2011 revealed complete resolution of hypermetabolic left lower lobe pulmonary nodules and osseous lesions, and near complete resolution of hypermetabolic liver metastases and neck masses (fig. 1b). Subsequently, she received a total of 3,750 cGy in 15 fractions via IMRT (with concurrent weekly chemotherapy) to the residual left neck mass. In addition to her radiographic 
response and resolution of cancer-related symptoms, her abnormal liver function tests, lactate dehydrogenase, and tumor markers also returned to normal range.

Pathologic Findings, Serum Tumor Marker Analysis and Molecular Analysis

A liver lesion biopsy demonstrated small cohesive clusters of atypical epithelial cells, predominately in portal areas, with rare foci of angiolymphatic involvement (fig. 2). Rare intracytoplasmic lumina were observed. The neoplastic cells were positive for cytokeratin 7 and OC125 (CA 125), while negative for cytokeratin 20, chromogranin, synaptophysin, estrogen receptor (ER), progesterone receptor (PR), thyroid transcription factor 1, CDX2, BRST-2 and HER2/neu. The neck mass biopsy sample also showed a tumor with a similar morphology and immunoprofile.

In order to identify potential therapeutic targets and biomarkers, further immunohistochemistry and molecular studies were performed. As demonstrated in fig. 3 an immunohistochemistry study showed high-level expression of the following: topoisomerase I (Topo I; 2+, 10\%); androgen receptor (AR; $1+, 20 \%)$, and ribonucleoside-diphosphate reductase large subunit $(2+, 80 \%)$. The tumor was found to be negative for thymidylate synthase (TS), Topo II- $\alpha$, and 06-methylguanine-DNA methyltransferase. Fluorescence in situ hybridization was performed with a probe specific for HER2/neu and a probe for the pericentromeric region of chromosome 17 (Vysis) showed no amplification of this gene.

Microarray analysis of RNA expression on formalin-fixed tissue was performed using the Pathwork ${ }^{\circledR}$ Tissue Of Origin (TO0) test (Pathwork Diagnostics, Sunnyvale, Calif., USA). The result showed that the tumor was consistent with breast primary, with a probability score of $98 \%$.

\section{Discussion}

We describe a 66-year-old female with metastatic adenocarcinoma of unknown primary who had a near complete response to second-line chemotherapy FOLFIRI, which was selected based on her tumor expression profile. The molecular profiling demonstrated that the primary tumor most likely originated from the breast. The absence of staining for ER, PR, and HER2/neu further classified her cancer as triplenegative breast cancer (TNBC).

CUP represents a real diagnostic problem and therapeutic challenge [1, 2]. Molecular profiling of tumors is a promising technique to improve the site of origin diagnosis in CUP patients [3]. When used in concert with clinical features and immunohistochemical stains, molecular profiling may provide the basis for more successful site-directed therapy for many patients [3-7]. A microarray-based gene expression diagnostic test, the Pathwork T00 test (Pathwork Diagnostics), analyzes the expression of 1,550 genes by applying normalization and classification algorithms to gene expression data from a microarray. In a blinded multicenter evaluation of 547 known primaries, the test exhibited an overall agreement of $87.8 \%$ with the pathologist-issued diagnosis [8]. In another retrospective cohort, the investigators evaluated the clinical utility of the Pathwork T00 test (Pathwork Diagnostics) in specimens from 21 patients diagnosed with CUP. In that cohort, the test demonstrated a probability to identify a single primary site in $16(76 \%)$ of the cases [9].

Traditionally, cisplatin-based chemotherapy regimens have been used to treat patients with CUP. Unfortunately, our patient had a stroke after 4 weeks of therapy. Cisplatin [10], her large tumor burden and high serum mucin [11] may have contributed to her CVA. Remarkably, she recovered without any residual neurological complications despite initial MRI findings. One of the most interesting aspects of this report was the correlation of the expression profiles with the therapeutic response of 
second-line chemotherapy. There is limited information on second-line therapy for CUP [2]. Empiric chemotherapy with carboplatin and paclitaxel would be less than optimal in patients with breast cancer. In this case, based on the tumor expression profile of a high expression of Topo I and a low level of TS, the patient was successfully treated with FOLFIRI, a combination regimen frequently used in metastatic colon cancer.

TNBC is a subtype of breast cancer that is refractory to some of the most effective therapies available for breast cancer treatment, including HER2-directed therapy and endocrine therapy [12]. Metastatic TNBC presents with higher rates of visceral metastases, has a relatively shorter median survival of 7-13 months, and has a limited duration of response to successive lines of chemotherapy (median response duration of 12 weeks to first line, 9 weeks to second line, and 4 weeks to third line) [13]. Clearly, there is an obvious need for alternative therapeutic strategies targeted to TNBC, as well as biomarkers that predict tumor response and resistance. Identification of genes differentially expressed in normal and tumor tissues may help in understanding tumor development, in discovering diagnostic and prognostic markers, and in identifying novel targets for drug therapy $[12,13]$. It has long been recognized that interpatient variability in response to chemotherapy is associated with different outcomes. The findings of overexpression of Topo I and underexpression of TS in the tumor sample, and the observed near complete clinical response with irinotecan and a fluoropyrimidine-based regimen in this case are interesting; they are consistent with previous reports in other cancers $[14,15]$. The activity of Topo inhibitors and the potential usefulness of Topo expression as a biomarker should be further tested in prospective studies. Another interesting observation is the overexpression of AR in our patient's tumor sample. The expression of AR was found in about $20 \%$ of patients with TNBC, which correlated with the aggressive biological behavior of the tumor [16, 17]. A phase II trial (NCT 004687150) is now testing the potential role of AR expression as a target for novel endocrinotherapy in TNBC [18].

The clinical assessment of response or failure to treatment usually requires a relatively long observation time. Tumor markers may provide early indication of effectiveness, thus driving cost-effective changes in therapeutic strategies in such a case. Recently, there has been a renewed interest towards tumor markers thanks to the meaningful changes in the management strategy of breast cancer patients. The high baseline level of the mucin 1 (MUC-1) serum marker at diagnosis and the correlation of tumor marker changes with clinical and radiographic response were noteworthy. The MUC-1 antigen is expressed in more than $90 \%$ of TNBC [19, 20], supporting the theory that MUC-1 may be a potentially useful biomarker as well as a target for a novel therapy. The MUC- 1 gene encodes a cell-associated mucin-like antigen, and different antibodies may be used to detect different epitopes. CA 15-3 and CA 27.29 are two related monoclonal antibodies that detect epitopes encoded by the MUC-1 gene [19, 20].

To summarize, we report an unusual case of CUP in a female patient who was successfully treated with second-line chemotherapy based on her tumor profile. Although no meaningful correlations or conclusions regarding the impact of the expression profile on efficacy of therapy can be drawn from a single case report, the usefulness of molecular profiling-based chemotherapy, and its impact on patients' outcome, should be tested further in future prospective clinical trials. 

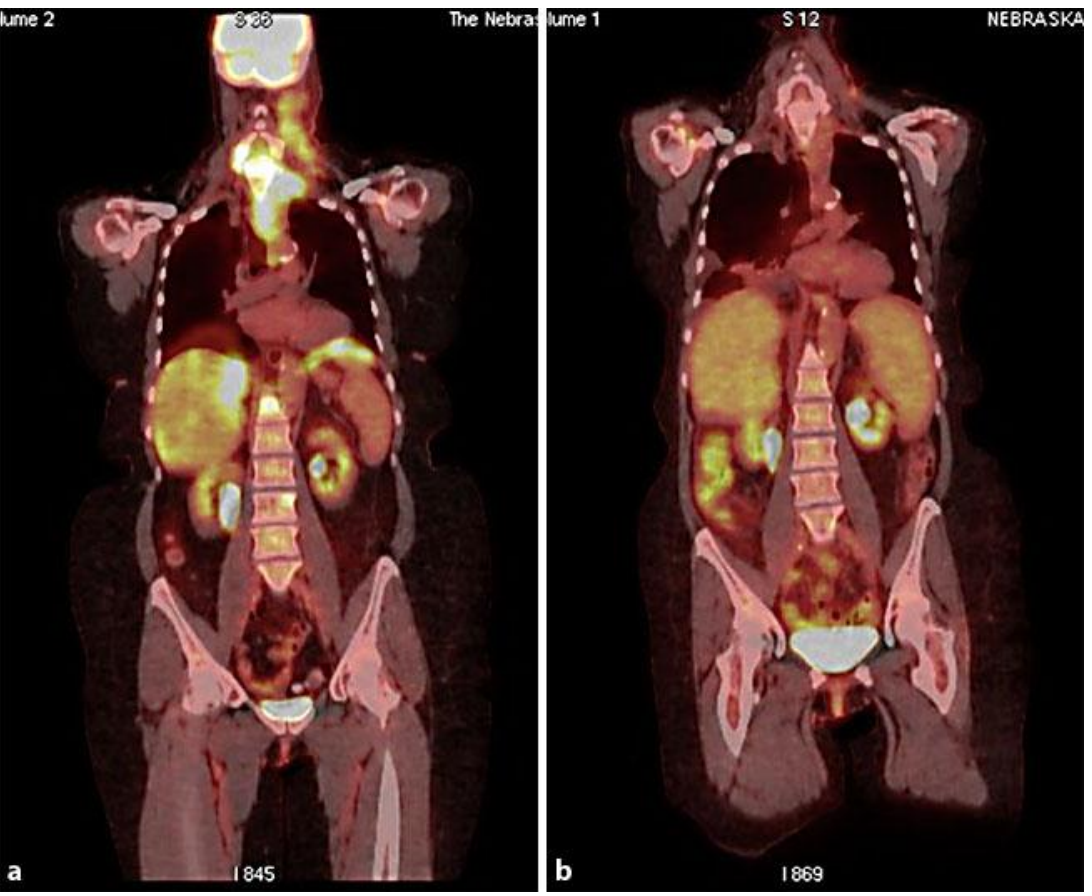

Fig. 1. FDG-PET/CT scan before (a) and after (b) chemotherapy. a Left level 3 large conglomerate mass (max. SUV of 10.5), left superior mediastinal large conglomerate mass (max. SUV of 7.8), numerous abnormal FDG uptakes involving skeletal structures, and multiple segmental liver abnormal FDG uptakes. b Markedly decreased hypermetabolic involvement of bulky masses involving the left neck, left cervical and left thoracic inlet. Near complete resolution of hypermetabolic liver metastases. Interval complete resolution of hypermetabolic left lower lobe pulmonary nodule and osseous lesions. 

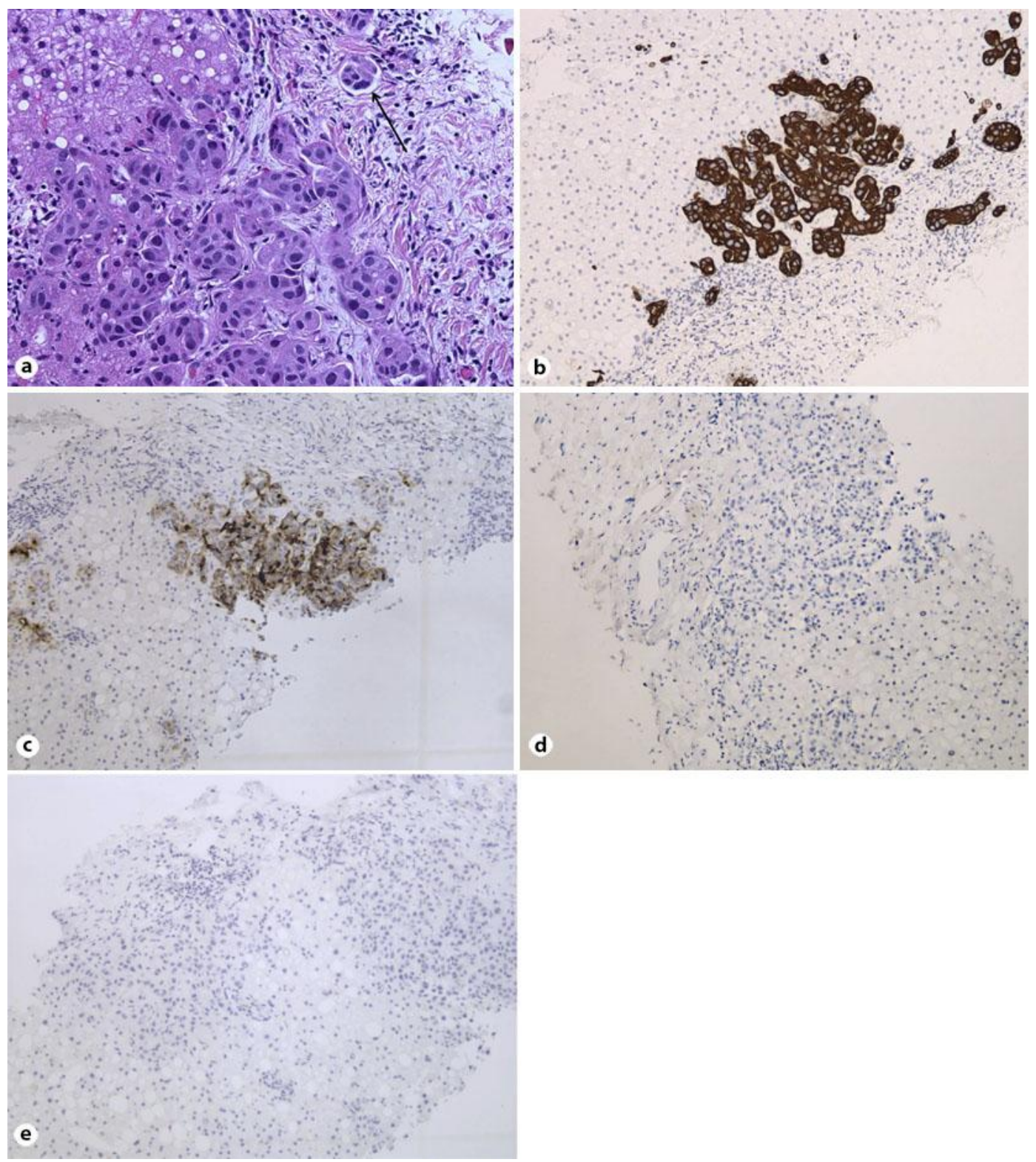

Fig. 2. H\&E sections from the liver lesion biopsy demonstrated clusters of neoplastic cells in portal areas with a focus of angiolymphatic invasion (arrow; a). The tumor cells are positive for CK7 (b) and 0C125 (c), while negative for estrogen receptor (d) and BRST-2 (e). 

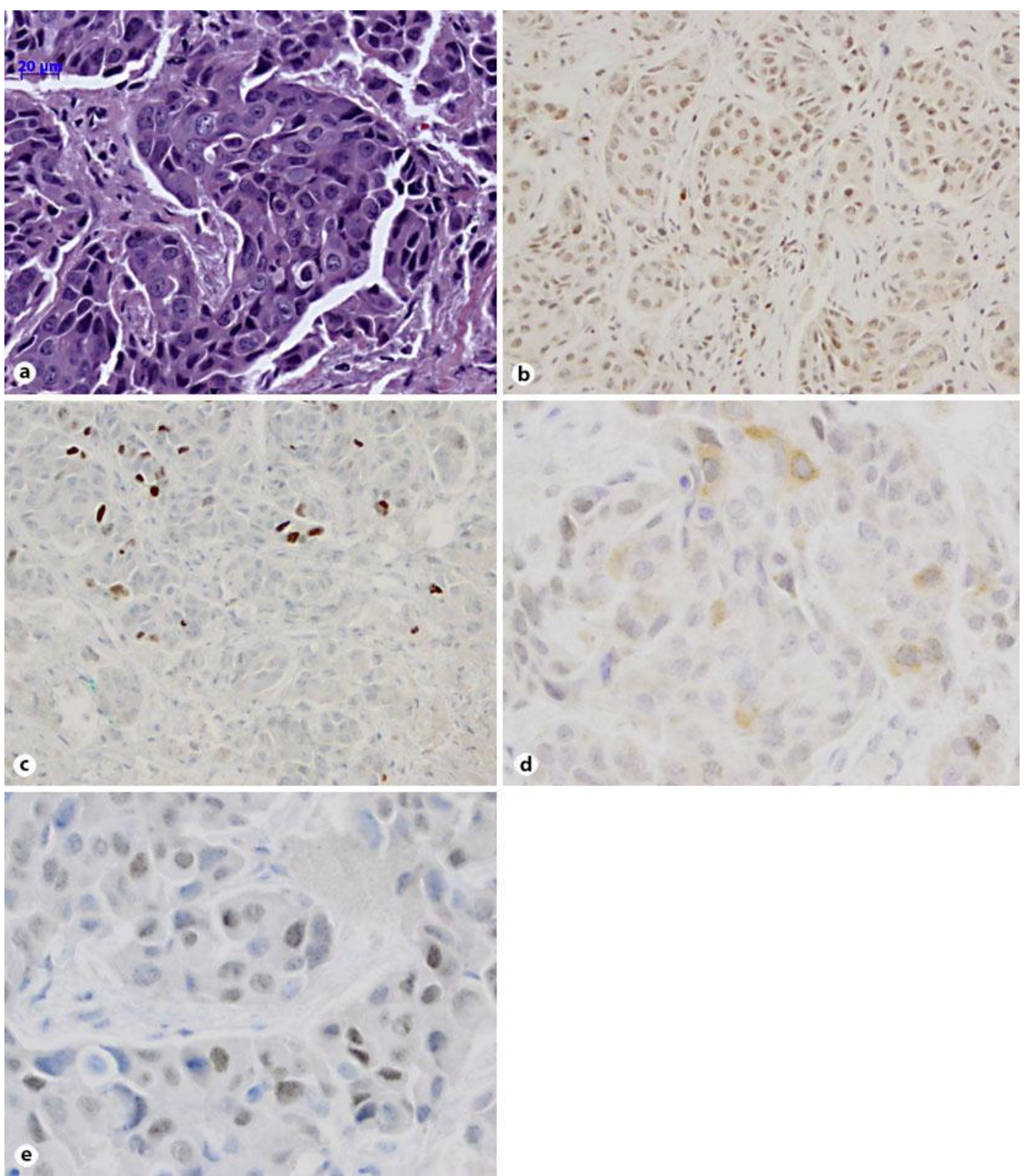

Fig. 3. Immunohistochemistry findings of the neck mass biopsy. H\&E sections of poorly differentiated adenocarcinoma (a), Topo I (b), Topo II- $\alpha$ (c), thymidylate synthase (d), and androgen receptor (e). 


\section{References}

1 Morris GJ, Greco FA, Hainsworth JD, Engstrom PF, Scialla S, Jordan WE 3rd, Thomas LC: Cancer of unknown primary site. Semin Oncol 2010;37:71-79.

-2 Greco FA, Hainsworth JD: Introduction: unknown primary cancer. Semin Oncol 2009;36:6-7.

-3 Horlings HM, van Laar RK, Kerst JM, Helgason HH, Wesseling J, van der Hoeven JJ, Warmoes MO, Floore A, Witteveen A, Lahti-Domenici J, Glas AM, Van't Veer LJ, de Jong D: Gene expression profiling to identify the histogenetic origin of metastatic adenocarcinomas of unknown primary. J Clin Oncol 2008;26:44354441.

4 Varadhachary GR, Talantov D, Raber MN, Meng C, Hess KR, Jatkoe T, Lenzi R, Spigel DR, Wang Y, Greco FA, Abbruzzese JL, Hainsworth JD: Molecular profiling of carcinoma of unknown primary and correlation with clinical evaluation. J Clin Oncol 2008;26:4442-4448.

5 Greco FA, Spigel DR, Yardley DA, Erlander MG, Ma XJ, Hainsworth JD: Molecular profiling in unknown primary cancer: accuracy of tissue of origin prediction. Oncologist 2010;15:500-506.

6 Greco FA, Erlander MG: Molecular classification of cancers of unknown primary site. Mol Diagn Ther 2009;13:367-373.

-7 Monzon FA, Medeiros F, Lyons-Weiler M, Henner WD: Identification of tissue of origin in carcinoma of unknown primary with a microarray-based gene expression test. Diagn Pathol 2010;5:3.

-8 Monzon FA, Lyons-Weiler M, Buturovic LJ, Rigl CT, Henner WD, Sciulli C, Dumur CI, Medeiros F, Anderson GG: Multicenter validation of a 1,550-gene expression profile for identification of tumor tissue of origin. J Clin Oncol 2009;27:2503-2508.

-9 Dumur CI, Lyons-Weiler M, Sciulli C, Garrett CT, Schrijver I, Holley TK, Rodriguez-Paris J, Pollack JR, Zehnder JL, Price M, Hagenkord JM, Rigl CT, Buturovic LJ, Anderson GG, Monzon FA: Interlaboratory performance of a microarray-based gene expression test to determine tissue of origin in poorly differentiated and undifferentiated cancers. J Mol Diagn 2008;10:67-77.

10 Saynak M, Cosar-Alas R, Yurut-Caloglu V, Caloglu M, Kocak Z, Uzal C: Chemotherapy and cerebrovascular disease. J BUON 2008;13:31-36.

11 Amico L, Caplan LR, Thomas C: Cerebrovascular complications of mucinouscancers. Neurology 1989;39:522-526.

12 Santana-Davila R, Perez EA: Treatment options for patients with triple-negative breast cancer. J Hematol Oncol 2010;3:42.

13 Kassam F, Enright K, Dent R, et al: Survival outcomes for patients with metastatic triple-negative breast cancer: implications for clinical practice and trial design. Clin Breast Cancer 2009;9:29-33.

$\checkmark 14$ Braun MS, Richman SD, Quirke P, et al: Predictive biomarkers of chemotherapy efficacy in colorectal cancer: results from the UK MRC FOCUS trial. J Clin Oncol 2008;26:2690-2698.

15 Paradiso A, Simone G, Petroni S, Leone B, et al: Thymidilate synthase and p53 primary tumour expression as predictive factors for advanced colorectal cancer patients. Br J Cancer 2000;82:560-567.

16 Luo X, Shi YX, Li ZM, Jiang WQ: Expression and clinical significance of androgen receptor in triple negative breast cancer. Chin J Cancer 2010;29:585-590.

-17 Doane AS, Danso M, Lal P, Donaton M, Zhang L, Hudis C, Gerald WL: An estrogen receptor-negative breast cancer subset characterized by a hormonally regulated transcriptional program and response to androgen. Oncogene 2006;25:3994-4008.

18 http://clinicaltrials.gov/ct2/show/NCT00468715

19 Cheung KL, Graves CRL, Robertson JFR: Tumour marker measurements in the diagnosis and monitoring of breast cancer. Cancer Treat Rev 2000;26:91-102.

20 Duffy MJ: CA 15-3 and related mucins as circulating markers in breast cancer. Ann Clin Biochem 1999;36:579-586. 